\title{
Fuzzy Homogeneous Bitopological Spaces
}

\author{
Samer Al Ghour, Almothana Azaizeh \\ ${ }^{1}$ Department of Mathematics and Statistics, Jordan University of Science and Technology, Jordan \\ ${ }^{2}$ College of Applied Studies and Community Service, Imam Abdurrahman Bin Faisal University, Saudi Arabia
}

\section{Article Info}

Article history:

Received Feb 15, 2018

Revised May 28, 2018

Accepted Jun 11, 2018

\section{Keyword:}

Cut topologies

Fuzzy bitopology

Fuzzy homeomorphism

Homogeneous topology

Minimal fuzzy open set

\begin{abstract}
We continue the study of the concepts of minimality and homogeneity in the fuzzy context. Concretely, we introduce two new notions of minimality in fuzzy bitopological spaces which are called minimal fuzzy open set and pairwise minimal fuzzy open set. Several relationships between such notions and a known one are given. Also, we provide results about the transformation of minimal, and pairwise minimal fuzzy open sets of a fuzzy bitopological space, via fuzzy continuous and fuzzy open mappings, and pairwise continuous and pairwise open mappings, respectively. Moreover, we present two new notions of homogeneity in the fuzzy framework. We introduce the notions of homogeneous and pairwise homogeneous fuzzy bitopological spaces. Several relationships between such notions and a known one are given. And, some connections between minimality and homogeneity are given. Finally, we show that cut bitopological spaces of a homogeneous (resp. pairwise homogeneous) fuzzy bitopological space are homogeneous (resp. pairwise homogeneous) but not conversely.
\end{abstract}

Copyright (C) 2018 Institute of Advanced Engineering and Science. All rights reserved.

\section{Corresponding Author:}

Samer Al Ghour,

Department of Mathematics and Statistics,

Jordan University of Science and Technology, Jordan.

Email: algore@just.edu.jo

\section{INTRODUCTION}

Throughout this paper, $I$ will denote the interval $[0,1]$. Let $X$ be a nonempty set. A member of $I^{X}$ is called a fuzzy subset of $X$ [1]. Throughout this paper, for $A, B \in I^{X}$ we write $A \leq B$ iff $A(x) \leq B(x)$ for all $x \in X$. By $A=B$ we mean that $A \leq B$ and $B \leq A$, i.e., $A(x)=B(x)$ for all $x \in X$. Also we write $A<B$ iff $A \leq B$ and $A \neq B$. If $\left\{A_{j}: j \in J\right\}$ is a collection of fuzzy sets in $X$, then $\left(V_{j}\right)(x)=\sup \left\{A_{j}(x): j \in\right.$ $J\}, x \in X$; and $\left(\wedge A_{j}\right)(x)=\inf \left\{A_{j}(x): j \in J\right\}, x \in X$.If $r \in[0,1]$ then $r_{X}$ denotes the fuzzy set given by $r_{X}(x)=r$ for all $x \in X$. If $U \subseteq X$ then $\mathcal{X}_{U}$ denotes the characteristic function of $U$. A fuzzy set $p$ defined by

$$
p(x)= \begin{cases}t, & \text { if } x=x_{p} \\ 0, & \text { if } x \neq x_{p}\end{cases}
$$

where $0<t \leq 1$ is called a fuzzy point in $X, x_{p} \in X$ is called the support of $p$ and $p\left(x_{p}\right)=t$ the value (level) of $p$ [2]. In this paper, a fuzzy point $p$ in $X$ is said to belong to a fuzzy set $A$ in $X$ [3] (notation: $p \in$ A) iff $p\left(x_{p}\right) \leq A\left(x_{p}\right)$.

Let $f: X \rightarrow Y$ be an ordinary mapping. We define

$$
f^{\rightarrow}: I^{X} \rightarrow I^{Y} \text { and } f^{\leftarrow}: I^{Y} \rightarrow I^{X}
$$


By

$$
\left(f^{\rightarrow}(A)\right)(y)= \begin{cases}\sup \left\{\mathrm{A}(\mathrm{x}): \mathrm{x} \in \mathrm{f}^{-1}(\{\mathrm{y}\}\},\right. & \text { if } y \in \text { range } f \\ 0, & \text { if } y \notin \text { range } f\end{cases}
$$

and $f^{\leftarrow}(B)=B \circ f$. A fuzzy topological space [4] is a pair $(X, \mathfrak{I})$, where $\mathrm{X}$ is a nonempty set, $\mathfrak{J}$ called a fuzzy topology on it is a subfamily of $I^{X}$ satisfying the following three axioms.

(1) $0_{X}, 1_{X} \in \mathfrak{J}$.

(2) If $A, B \in \mathfrak{I}$, then $A \wedge B \in \mathfrak{I}$.

(3) If $\left\{A_{j}: j \in J\right\} \subseteq \mathfrak{I}$, then $\vee\left\{A_{j}: j \in J\right\} \in \mathfrak{J}$.

Let $(X, \mathfrak{I})$ be a fuzzy topological space, $\mathfrak{I}_{\circ} \subseteq \mathfrak{I}$. $\mathfrak{I}_{0}$ is called a base of $\mathfrak{I}$, if $\mathfrak{I}=\left\{\bigvee A: A \subseteq \mathfrak{I}_{0}\right\} \cup$ $\left\{0_{X}\right\} . \mathfrak{I}_{0}$ is called a subbase of $\mathfrak{I}$, if $\left\{\wedge A: A \subseteq \mathfrak{I}_{0}\right.$ and $A$ is a nonempty finite set $\}$ forms a base of $\mathfrak{J}$. Let $f:\left(X, \mathfrak{I}_{1}\right) \rightarrow\left(Y, \mathfrak{\Im}_{2}\right)$ be a function. $\mathrm{f}$ is fuzzy continuous [2] if $f^{\leftarrow}(B) \in \mathfrak{J}_{1}$ for all $B \in \mathfrak{I}_{2} . f$ is fuzzy open [2] if $\left(f^{\rightarrow}(A) \in \mathfrak{I}_{2}\right.$ for all $A \in \mathfrak{I}_{1}$. $\mathrm{f}$ is fuzzy homeomorphism if $f$ is bijective, fuzzy continuous and fuzzy open. In 1963, Kelly [5] introduced the notion of bitopological spaces as an ordered triple $\left(X, \tau_{1}, \tau_{2}\right)$ of a set $X$ and two topologies $\tau_{1}$ and $\tau_{2}$, (i.e., two bitopological spaces $\left(X, \tau_{1}, \tau_{2}\right)$ and $\left(X, \tau_{1}{ }^{\prime}, \tau_{2}{ }^{\prime}\right)$ are identical if and only if $\tau_{i}=\tau_{i}{ }^{\prime}$ for each $i \in\{1,2\}$ and similarly, the author in [6], defined the notion of fuzzy bitopological spaces. The area of research in fuzzy bitopological spaces is still a very hot research topic [7-9].

The authors in [10] introduced the concept of homogeneous fuzzy topological space as follows: A fuzzy topological space $(X, \Im)$ is called homogeneous if for any two points $x, y \in X$, there exists a fuzzy homeomorphism $h:(X, \mathfrak{I}) \rightarrow(X, \mathfrak{J})$ such that $h(x)=y$. A nonempty open set $M$ of an ordinary topological space $(X, \tau)$ is called a minimal open set in $X$ [11] if any open set in $X$ which is contained in $M$ is $\varnothing$ or $M$. The authors in [12] extended the concept minimal open set to include fuzzy topological spaces as follows: A fuzzy open set $A$ of a fuzzy topological space $(X, \mathfrak{J})$ is called a minimal fuzzy open set in $X$ if $A$ is nonzero and there is no nonzero fuzzy open set $B$ such that $B<A$, and then Al Ghour continued the study of minimal fuzzy open sets in $[13,14]$. Recently, the authors in $[15]$ introduced and investigated two types of minimal open sets in bitopological spaces and using them they obtained some homogeneity results in bitopological spaces. As defined in [16], for fuzzy topological space $(X, \mathfrak{J})$, the associated topological space $\left\{B^{-1}(a, 1]: B \in \mathfrak{J}\right\}$ is called the $a$-cut (level) topological space and denoted by $\mathfrak{I}_{a}$. Cut topological spaces have been studied in deep by a number of authors. Some authors were used cut topological spaces for solving some problems of fuzzy topology by reducing them to standard problems of general topology (see [17-26]). Also cut topological spaces have shown to be useful in fuzzy automata theory in [27-29].

In this paper the we continue the study of the concepts of minimality and homogeneity in the fuzzy context. Concretely, in Section 2 we introduce two new notions of minimality in fuzzy bitopological spaces which are called minimal fuzzy open set and pairwise minimal fuzzy open set. Several relationships between such notions and a known one are given in Theorem 2.2, Theorem 2.5, Theorem 2.8 and Theorem 2.10. Moreover, in the same section, we provide results about the transformation of minimal, and pairwise minimal fuzzy open sets of a fuzzy bitopological space, via fuzzy continuous and fuzzy open mappings, and pairwise continuous and pairwise open mappings, respectively. Section 3 is devoted to present two new notions of homogeneity in the fuzzy framework. In fact, we introduce the notions of homogeneous and pairwise homogeneous fuzzy bitopological spaces. Several relationships between such notions and a known one are given in Theorem 3.3., Theorem 3.7 and Corollary 3.8. Moreover, some connections between minimality and homogeneity are given in Theorem 3.9, Theorem 3.10 and Theorem 3.11. Section 4 is devoted to show that cut bitopological spaces of a homogeneous (resp. pairwise homogeneous) fuzzy bitopological space are homogeneous (resp. pairwise homogeneous) but not conversely. The following definitions and results will be used in the sequel.

Definition 1.1. Let $\mathfrak{I}_{1}$ and $\mathfrak{I}_{2}$ be two fuzzy topologies on a nonempty set $X$. Then $\mathfrak{I}_{1} \cup \mathfrak{I}_{2}$ forms a subbase for some fuzzy topology on $X$. This fuzzy topology is called the least upper bound fuzzy topology on $\mathrm{X}$ and denoted by $\left\langle\mathfrak{I}_{1}, \mathfrak{I}_{2}\right\rangle$. It is clear that each basic fuzzy open set in $\left\langle\mathfrak{I}_{1}, \mathfrak{I}_{2}\right\rangle$ can be written in the form $\mathrm{A} \wedge \mathrm{B}$ where $\mathrm{A} \in \mathfrak{I}_{1}$ and $B \in \mathfrak{I}_{2}$.

Definition 1.2. Let $f:\left(X, \mathfrak{J}_{1}, \mathfrak{I}_{2}\right) \rightarrow\left(Y, \delta_{1}, \delta_{2}\right)$ be a function.

a. $\quad f$ is called fuzzy continuous (fuzzy open, fuzzy homeomorphism) iff the functions $f:\left(X, \widetilde{\Im}_{1}\right) \rightarrow\left(Y, \delta_{1}\right)$ and $f:\left(X, \mathfrak{J}_{2}\right) \rightarrow\left(Y, \delta_{2}\right)$ are fuzzy continuous (fuzzy open, fuzzy homeomorphism respectively).

b. $\quad f$ is called fuzzy pairwise continuous iff for each $B \in \delta_{1} \cup \delta_{2}, f^{\leftarrow}(B) \in \mathfrak{I}_{1} \cup \mathfrak{J}_{2}$.

c. $\quad f$ is called fuzzy pairwise homeomorphism iff $f$ is a bijection, fuzzy pairwise continuous and $f^{-1}:\left(Y, \delta_{1}, \delta_{2}\right) \rightarrow\left(X, \widetilde{J}_{1}, \mathfrak{\Im}_{2}\right)$ is fuzzy pairwise continuous.

Proposition 1.3. [12] Let $f:\left(X, \widetilde{I}_{1}\right) \rightarrow\left(Y, \widetilde{I}_{2}\right)$ be a fuzzy continuous and fuzzy open function. If $A$ is a minimal fuzzy open set in $\left(X, \mathfrak{I}_{1}\right)$ then $\left(f^{\rightarrow}(A)\right.$ is a minimal fuzzy open set in $\left(Y, \mathfrak{\Im}_{2}\right)$. 
Proposition 1.4. [12] Let $(X, \mathfrak{I})$ be a homogeneous fuzzy topological space which contains a minimal fuzzy open set. Then we have the following.

a. The collection of all minimal fuzzy open sets in $(X, \mathfrak{J})$ can be written of the form $\left\{r \mathcal{X}_{G_{\alpha}}: \alpha \in \Lambda\right\}$ where $r \in(0,1]$ and $\left\{G_{\alpha}: \alpha \in \Lambda\right\}$ is a partition on $\mathrm{X}$ and $\left|G_{\alpha}\right|=\left|G_{\beta}\right|$ for all $\alpha, \beta \in \Lambda$.

b. For any fuzzy open set $A$ in $X$ and $\alpha \in \Lambda$ either $\left.A\right|_{G_{\alpha}}=0$ or $A(x) \geq r$ for all $x \in G_{\alpha}$.

Definition 1.5. [15] Let $f:\left(X, \tau_{1}, \tau_{2}\right) \rightarrow\left(Y, \sigma_{1}, \sigma_{2}\right)$ between bitopological spaces.

a. $\quad f$ is called continuous (open, homeomorphism) iff the functions $f:\left(X, \tau_{1}\right) \rightarrow\left(Y, \sigma_{1}\right)$ and $f:\left(X, \tau_{2}\right) \rightarrow$ $\left(Y, \sigma_{2}\right)$ are continuous (open, homeomorphism respectively).

b. $\quad \mathrm{f}$ is called pairwise continuous iff for each $V \in \sigma_{1} \cup \sigma_{2}, f^{-1}(V) \in \tau_{1} \cup \tau_{2}$.

c. $\quad f$ is called pairwise homeomorphism iff $f$ is a bijection, pairwise continuous and $f^{-1}:\left(Y, \sigma_{1}, \sigma_{2}\right) \rightarrow$ $\left(X, \tau_{1}, \tau_{2}\right)$ is pairwise continuous.

Definition 1.6. [15] A bitopological space $\left(X, \tau_{1}, \tau_{2}\right)$ is said to be homogeneous (resp. pairwise homogeneous) if for any two points $x_{1}, x_{2} \in X$ there exists a homeomorphism (resp. pairwise homeomorphism) $h:\left(X, \tau_{1}, \tau_{2}\right) \rightarrow\left(Y, \sigma_{1}, \sigma_{2}\right)$ such that $h\left(x_{1}\right)=x_{2}$.

\section{MINIMALITY IN FUZZY BITOPOLOGICAL SPACES}

Definition 2.1. Let $\left(X, \mathfrak{I}_{1}, \mathfrak{I}_{2}\right)$ be a fuzzy bitopological space.

a. $\quad$ A fuzzy set $A$ of $X$ is called a minimal fuzzy open set in $\left(X, \mathfrak{J}_{1}, \mathfrak{I}_{2}\right)$ if $A$ is a minimal fuzzy open set in both of $\left(X, \mathfrak{J}_{1}\right)$ and $\left(X, \mathfrak{J}_{2}\right)$.

b. A nonzero fuzzy set $A$ of $X$ is called a pairwise minimal fuzzy open set in $\left(X, \mathfrak{J}^{1}, \mathfrak{J}^{2}\right)$ if $A \in \mathfrak{J}^{1} \cup \mathfrak{I}^{2}$ and for any fuzzy set $B \in \mathfrak{J}^{1} \cup \mathfrak{I}^{2}$ with $B \leq A, B=0_{X}$ or $B=A$.

As a simple example of a fuzzy bitopological space that have a minimal fuzzy open set, take $X=$ $\{a, b, c\}, \widetilde{I}_{1}=\left\{0_{X}, 1_{X}, \mathcal{X}_{\{a\}}, \mathcal{X}_{\{b\}}, \mathcal{X}_{\{a, b\}}\right\}, \mathfrak{I}_{2}=\left\{0_{X}, 1_{X}, \mathcal{X}_{\{a\}}, \mathcal{X}_{\{c\}}, \mathcal{X}_{\{a, c\}}\right\}$, it is clear that $\mathcal{X}_{\{a\}}$ is a minimal fuzzy open set in $\left(X, \mathfrak{J}_{1}, \mathfrak{I}_{2}\right)$. Theorem 2.2 . Given a fuzzy bitopological space $\left(X, \mathfrak{J}_{1}, \mathfrak{I}_{2}\right)$ and $A$ be a fuzzy set of $X$. Consider the following statements.

(a) $A$ is a minimal fuzzy open set in $\left(X, \mathfrak{I}_{1}, \mathfrak{I}_{2}\right)$.

(b) $A$ is a minimal fuzzy open set in $\left(X,\left\langle\mathfrak{I}_{1}, \mathfrak{I}_{2}\right\rangle\right)$ with $A \in \mathfrak{I}_{1} \cup \mathfrak{I}_{2}$.

(c) $A$ is a pairwise minimal fuzzy open set in $\left(X, \mathfrak{I}_{1}, \mathfrak{I}_{2}\right)$.

Then $(a) \Rightarrow(b) \Rightarrow(c)$.

Proof.

a. $\quad(a) \Rightarrow(b)$ Since $A$ is a minimal fuzzy open set in $\left(X, \mathfrak{I}_{1}, \mathfrak{I}_{2}\right)$, then $A \in \mathfrak{J}_{1} \cap \mathfrak{I}_{2} \subseteq \mathfrak{J}_{1} \cup \mathfrak{I}_{2} \subseteq\left\langle\mathfrak{I}_{1}, \mathfrak{I}_{2}\right\rangle$. Suppose for some nonzero fuzzy set $B \in\left\langle\mathfrak{I}_{1}, \mathfrak{I}_{2}\right\rangle$ we have $B \leq A$. Choose $x_{0} \in X$ such that $B\left(x_{0}\right)>0$. Consider the fuzzy point $p$ with support $x_{p}=x_{0}$ and level $p\left(x_{p}\right)=B\left(x_{0}\right) / 2$. Then $p \in B$ and so there exists a fuzzy set $C \wedge D$ where $C \in \mathfrak{I}_{1}, B \in \mathfrak{I}_{2}, p \in C \wedge D$, and $C \wedge D \leq B$. Since $A$ is a minimal fuzzy open set in $\left(X, \mathfrak{J}_{1}, \mathfrak{J}_{2}\right)$ then $A \leq C$ and $A \leq D$ and hence $A \leq C \wedge D \leq B$. Therefore, $A=B$.

b. $\quad(b) \Rightarrow(c)$ Suppose for some nonzero $B \in \mathfrak{I}^{1} \cup \mathfrak{I}^{2}, B \leq A$. Since $\mathfrak{I}^{1} \cup \mathfrak{I}^{2} \subseteq\left\langle\mathfrak{I}^{1}, \mathfrak{J}^{2}\right\rangle$, it follows that $B=A$. The following example clarifies in Theorem 2.2 that $(c) \nRightarrow(b)$.

Example 2.3. Let $X=\{a, b\}$ with the fuzzy topologies $\mathfrak{I}_{1}=\left\{0_{X}, 1_{X}, A\right\}$ and $\mathfrak{I}_{2}=\left\{0_{X}, 1_{X}, B\right\}$ where $A=$ $\{(a, 0.5),(b, 0.25)\}$ and $B=\{(a, 0),(b, 0.5)\}$. Note that $A \wedge B \in\left\langle\mathfrak{I}_{1}, \mathfrak{I}_{2}\right\rangle, A \wedge B \neq 0 \_\{X\}$, and $A \wedge B<A$. Therefore, $\mathrm{A}$ is a pairwise minimal fuzzy open set in $\left(X, \mathfrak{J}_{1}, \mathfrak{I}_{2}\right)$ but not a minimal fuzzy open set in $\left(X,\left\langle\mathfrak{I}_{1}, \mathfrak{I}_{2}\right\rangle\right)$. The following example will show in Theorem 2.2 that $(b) \nRightarrow(a)$.

Example 2.4. Let $X=\{a, b\}$ with the fuzzy topologies $\mathfrak{J}_{1}=\left\{0_{X}, 1_{X}, A\right\}$ where $A=\{(a, 0),(b, 0.5)\}$ and $\mathfrak{I}_{2}=\left\{0_{X}, 1_{X}\right\}$. Then $A$ is a pairwise minimal fuzzy open set in $\left(X, \mathfrak{J}_{1}, \mathfrak{I}_{2}\right)$ with $A \in \mathfrak{I}_{1} \cup \mathfrak{I}_{2}$ but not a minimal fuzzy open set in $\left(X, \mathfrak{I}_{1}, \mathfrak{J}_{2}\right)$.

In Theorem 2.2, if we add the condition " $A \in \mathfrak{I}_{1} \cap \mathfrak{I}_{2}$ ", then converse of each implication will be true. Theorem 2.5. Let $\left(X, \mathfrak{J}_{1}, \mathfrak{I}_{2}\right)$ be a fuzzy bitopological space and $A \in \mathfrak{I}_{1} \cap \mathfrak{I}_{2}$. Then the following are equivalent.

(a) $A$ is a minimal open set in $\left(X, \mathfrak{J}_{1}, \mathfrak{I}_{2}\right)$.

(b) $A$ is a minimal open set in $\left(X,\left\langle\mathfrak{I}_{1}, \mathfrak{I}_{2}\right\rangle\right)$ with $A \in \mathfrak{I}_{1} \cup \mathfrak{I}_{2}$.

(c) $A$ is a pairwise minimal open set in $\left(X, \mathfrak{I}_{1}, \mathfrak{I}_{2}\right)$.

Proof.

a. $\quad(a) \Rightarrow(b)$ and $(b) \Rightarrow(c)$ follow from Theorem 2.2.

b. $\quad(c) \Rightarrow(a)$ Without loss of generality we show that $A$ is a minimal fuzzy open set in $\left(X, \mathfrak{J}_{1}\right)$. 
Let $B \in \mathfrak{I}_{1}$ be nonzero with $B \leq A$. Since $B \in \mathfrak{I}_{1} \cup \mathfrak{I}_{2}$ and $A$ is a pairwise minimal fuzzy open set in $\left(X, \mathfrak{\Im}_{1}, \mathfrak{\Im}_{2}\right)$, then $B=A$. Theorem 2.6. If $A$ is a minimal fuzzy open set and $\mathrm{B}$ is a pairwise minimal fuzzy open set in a fuzzy bitopological space $\left(X, \mathfrak{J}_{1}, \mathfrak{I}_{2}\right)$, then either $A \wedge B=0_{X}$ or $A=B$.

Proof. Suppose $A \wedge B \neq 0_{X}$. From the assumptions, we conclude that $A \wedge B \in \mathfrak{I}_{1} \cup \mathfrak{I}_{2}$. Then we have the following cases:

a. Case 1: If $A \wedge B \in \mathfrak{I}_{1}$, then we conclude that $A=A \wedge B$, i.e., $A \leq B$, because $A$ is minimal fuzzy open in $\left(X, \widetilde{I}_{1}\right)$ and $A \wedge B \leq A$. Therefore, as $B$ is pairwise minimal fuzzy open in $\left(X, \widetilde{J}_{1}, \mathfrak{I}_{2}\right)$ and $A \in \mathfrak{I}_{1} \cup$ $\mathfrak{I}_{2}$, we conclude that $B=A$.

b. Case 2: If $A \wedge B \in \mathfrak{I}_{2}$, then we conclude that $A=A \wedge B$, i.e., $A \leq B$, because $A$ is minimal fuzzy open in $\left(X, \mathfrak{J}_{2}\right)$ and $A \wedge B \leq A$. Therefore, as $B$ is pairwise minimal fuzzy open in $\left(X, \mathfrak{J}_{1}, \mathfrak{J}_{2}\right)$ and $A \in \mathfrak{I}_{1} \cup$ $\Im_{2}$, we conclude that $B=A$.

Therefore, in both cases we show that $A=B$. Corollary 2.7. (i) Let $\left(X, \widetilde{J}_{1}, \widetilde{I}_{2}\right)$ be a fuzzy bitopological space. If $A$ and $B$ are two minimal fuzzy open sets in $\left(X, \mathfrak{\Im}_{1}, \mathfrak{J}_{2}\right)$, then either $A \wedge B=0_{X}$ or $A=B$. (ii) [9] Let $(X, \mathfrak{J})$ be a fuzzy topological space. If $A$ and $B$ are two minimal fuzzy open sets in $(X, \mathfrak{J})$, then either $A \wedge$ $B=0_{X}$ or $A=B$. Proof. (i) (resp. (ii)) is shown by Theorems 2.2 and 2.6 (resp. (i) above; take $\mathfrak{J}_{1}=\mathfrak{J}_{2}$ ).

In Example 2.3, A and B are pairwise minimal fuzzy open sets in $\left(X, \mathfrak{I}_{1}, \mathfrak{I}_{2}\right)$, but neither $A \wedge B=$ $0_{X}$ nor $A=B$. Therefore, in Theorem 2.6 we cannot replace minimality by pairwise minimality.

Theorem 2.8. If $A$ is a pairwise minimal fuzzy open set in a fuzzy bitopological space $\left(X, \mathfrak{J}_{1}, \mathfrak{I}_{2}\right)$ with $A \in \mathfrak{I}_{i}$ for some $i \in\{1,2\}$, then $A$ is a minimal fuzzy open set in $\left(X, \mathfrak{I}_{i}\right)$.

Proof. For each nonzero fuzzy set $B \in \mathfrak{I}_{i}$ with $B \leq A$, we have $B \in \mathfrak{I}_{1} \cup \mathfrak{I}_{2}$ and so $B=A$, hence $A$ is a minimal fuzzy open set in the fuzzy topological space $\left(X, \widetilde{I}_{i}\right)$.

Corollary 2.9. If $A$ is a pairwise minimal fuzzy open set in a fuzzy bitopological space $\left(X, \widetilde{J}_{1}, \widetilde{I}_{2}\right)$, then $A$ is a minimal fuzzy open set in $\left(X, \mathfrak{J}_{1}\right)$ or $A$ is a minimal fuzzy open set in $\left(X, \widetilde{J}_{2}\right)$.

Theorem 2.10. Let $A, B$ be two pairwise minimal fuzzy open sets in a fuzzy bitopological space $\left(X, \mathfrak{I}_{1}, \mathfrak{I}_{2}\right)$ with $A \neq B$ and $A \wedge B \neq 0_{X}$. Then $A \wedge B$ is a minimal fuzzy open set in $\left(X,<\mathfrak{J}_{1}, \mathfrak{J}_{2}>\right)$.

Proof. If $\{A, B\} \subseteq \mathfrak{I}_{i}$ where $i=1$ or $i=2$, then by Theorem 2.8, it follows that both $A$ and $B$ are minimal fuzzy open sets in $\left(X, \mathfrak{\Im}_{i}\right)$ and by Corollary 2.7 (ii), it follows that either $A=B$ or $A \wedge B=0_{X}$. Therefore, we may assume that $A \in \widetilde{I}_{1}$ and $B \in \mathfrak{I}_{2}$. Suppose there exists a nonzero fuzzy open set $W \in<$ $\mathfrak{I}_{1}, \mathfrak{I}_{2}>$ such that $W \leq A \wedge B$. Choose $U \in \mathfrak{I}_{1}$ and $V \in \widetilde{I}_{2}$ such that $0_{X} \neq U \wedge V \leq W \leq A \wedge B$. Choose a fuzzy point $q \in U \wedge V$. Then $q \in U \wedge A \in \widetilde{J}_{1}$ and $q \in V \wedge B \in \mathfrak{\Im}_{2}$. Thus, $U \wedge A=A$ and $V \wedge B=B$. Hence, $A \wedge B \leq U \wedge V \leq W$. This completes the proof.

Theorem 2.11. (i) Let $f:\left(X, \mathfrak{J}_{1}, \mathfrak{J}_{2}\right) \rightarrow\left(Y, \delta_{1}, \delta_{2}\right)$ be fuzzy continuous and fuzzy open function. If $A$ is a minimal fuzzy open set in $\left(X, \mathfrak{\Im}_{1}, \mathfrak{\Im}_{2}\right)$, then $f^{\rightarrow}(A)$ is a minimal fuzzy open set of $\left(Y, \delta_{1}, \delta_{2}\right)$. (ii) Let $f:\left(X, \mathfrak{I}_{1}, \mathfrak{I}_{2}\right) \rightarrow\left(Y, \delta_{1}, \delta_{2}\right)$ be a fuzzy homeomorphism. Then $A$ is a minimal fuzzy open set in $\left(X, \mathfrak{I}_{1}, \mathfrak{I}_{2}\right)$ if and only if $f^{\rightarrow}(A)$ is a minimal fuzzy open set in $\left(Y, \delta_{1}, \delta_{2}\right)$.

Proof. (i) Proposition 1.3. (ii) Follows from (i) above.

Theorem 2.12. Let $f:\left(X, \mathfrak{\Im}_{1}, \mathfrak{J}_{2}\right) \rightarrow\left(Y, \delta_{1}, \delta_{2}\right)$ be injective, fuzzy pairwise continuous, and fuzzy pairwise open function. If $A$ is a pairwise minimal fuzzy open set in $\left(X, \mathfrak{J}_{1}, \mathfrak{J}_{2}\right)$, then $f^{\rightarrow}(A)$ is a pairwise minimal fuzzy open set of $\left(Y, \delta_{1}, \delta_{2}\right)$.

Proof. Since $A$ is a pairwise minimal fuzzy open set then $A \neq 0_{X}$, and so $f^{\rightarrow}(A) \neq 0_{Y}$. Also, since $f$ is fuzzy pairwise open then $f^{\rightarrow}(A) \in \delta_{1} \cup \delta_{2}$. Suppose for some nonzero fuzzy set $B \in \delta_{1} \cup \delta_{2}, B \leq$ $f^{\rightarrow}(A)$. Then $f^{\leftarrow}(B) \leq f^{\leftarrow}\left(f^{\rightarrow}(A)\right)$. Since $f$ is injective, we have $f^{\leftarrow}\left(f^{\rightarrow}(A)\right)=A$. Choose $y_{0} \in Y$ such that $B\left(y_{0}\right)>0$. Since $B \leq f^{\rightarrow}(A)$, then $0<B\left(y_{0}\right) \leq\left(f^{\rightarrow}(A)\right)\left(y_{0}\right)=\sup \left\{A(x): f(x)=y_{0}\right\}$ and so there exists $x_{0} \in X$ such that $f\left(x_{0}\right)=y_{0}$. Therefore, $\left(f^{\leftarrow}(B)\right)\left(x_{0}\right)=B\left(y_{0}\right)>0$ and hence $f^{\leftarrow}(B) \neq 0_{X}$. Since $f$ is fuzzy pairwise continuous, then $f^{\leftarrow}(B) \in \mathfrak{J}_{1} \cup \mathfrak{J}_{2}$. Since $A$ is a pairwise minimal fuzzy open set, it follows that $A=f^{\leftarrow}(B)$. Hence, $f^{\wedge}\{\rightarrow\}(A)=f^{\rightarrow}\left(f^{\leftarrow}(B)\right) \leq B$. This ends the proof. Corollary 2.13. Let $f:\left(X, \widetilde{\Im}_{1}, \widetilde{J}_{2}\right) \rightarrow\left(Y, \delta_{1}, \delta_{2}\right)$ be a fuzzy pairwise homeomorphism. Then $A$ is a pairwise minimal fuzzy open set in $\left(X, \mathfrak{I}_{1}, \mathfrak{I}_{2}\right)$ if and only if $f^{\rightarrow}(A)$ is a pairwise minimal fuzzy open set of $\left(Y, \delta_{1}, \delta_{2}\right)$.

\section{PAIRWISE HOMOGENEITY IN FUZZY BITOPOLOGICAL SPACES}

Definition 3.1. A fuzzy bitopological space $\left(X, \widetilde{J}_{1}, \mathfrak{I}_{2}\right)$ is said to be homogeneous (resp. pairwise homogeneous) if for any two points $x_{1}, x_{2} \in X$ there exists a fuzzy homeomorphism (resp. fuzzy pairwise homeomorphism $) h:\left(X, \mathfrak{I}_{1}, \mathfrak{I}_{2}\right) \rightarrow\left(X, \mathfrak{\Im}_{1}, \mathfrak{I}_{2}\right)$ such that $h\left(x_{1}\right)=x_{2}$. Lemma 3.2. Let $f:\left(X, \mathfrak{I}_{1}, \mathfrak{\Im}_{2}\right) \rightarrow$ $\left(Y, \delta_{1}, \delta_{2}\right)$ be a function. (a) If $f:\left(X, \mathfrak{\Im}_{1}, \mathfrak{I}_{2}\right) \rightarrow\left(Y, \delta_{1}, \delta_{2}\right)$ is fuzzy pairwise continuous, then $f:(X,<$ 
$\left.\mathfrak{I}_{1}, \mathfrak{I}_{2}>\right) \rightarrow\left(Y,<\delta_{1}, \delta_{2}>\right)$ is fuzzy continuous. (b) If $f:\left(X, \mathfrak{I}_{1}, \mathfrak{I}_{2}\right) \rightarrow\left(Y, \delta_{1}, \delta_{2}\right)$ is fuzzy continuous, then $f:\left(X, \mathfrak{\Im}_{1}, \widetilde{J}_{2}\right) \rightarrow\left(Y, \delta_{1}, \delta_{2}\right)$ is fuzzy pairwise continuous. Proof. (a) For every $A \in \delta_{1}$ and $B \in \delta_{2}$, we have $\{A, B\} \subseteq \delta_{1} \cup \delta_{2}$ and $f^{\leftarrow}(A \wedge B)=f^{\leftarrow}(A) \wedge f^{\leftarrow}(B)$ where $\left\{f^{\leftarrow}(A), f^{\leftarrow}(B)\right\} \subseteq \Im_{1} \cup \Im_{2} \subseteq<\Im_{1}, \Im_{2}>$. This ends the proof. (b) Let $B \in \delta_{1} \cup \delta_{2}$. Without loss of generality we may assume that $B \in \delta_{1}$. As $f:\left(X, \mathfrak{I}_{1}, \mathfrak{I}_{2}\right) \rightarrow\left(Y, \delta_{1}, \delta_{2}\right)$ is fuzzy continuous, then $f:\left(X, \mathfrak{I}_{1}\right) \rightarrow\left(Y, \delta_{1}\right)$ is fuzzy continuous and hence $f^{\leftarrow}(B) \in \mathfrak{I}_{1} \subseteq \mathfrak{I}_{1} \cup \mathfrak{I}_{2}$

Theorem 3.3. Let $\left(X, \mathfrak{I}_{1}, \mathfrak{I}_{2}\right)$ be fuzzy bitopological space.

(a) If $\left(X, \mathfrak{I}_{1}, \mathfrak{I}_{2}\right)$ is pairwise homogeneous, then $\left(X,<\mathfrak{I}_{1}, \mathfrak{I}_{2}>\right)$ is homogeneous.

(b) If $\left(X, \mathfrak{I}_{1}, \mathfrak{I}_{2}\right)$ is homogeneous, then $\left(X, \mathfrak{I}_{1}, \mathfrak{I}_{2}\right)$ is pairwise homogeneous.

(c) If $\left(X, \mathfrak{I}_{1}, \mathfrak{I}_{2}\right)$ is homogeneous, then $\left(X, \mathfrak{\Im}_{1}\right)$ and $\left(X, \mathfrak{I}_{2}\right)$ are homogeneous.

Proof. (a) Let $x_{1}, x_{2} \in X$. As $\left(X, \mathfrak{I}_{1}, \mathfrak{I}_{2}\right)$ is pairwise homogeneous, there exists a fuzzy pairwise homeomorphism $h:\left(X, \mathfrak{I}_{1}, \mathfrak{I}_{2}\right) \rightarrow\left(X, \mathfrak{I}_{1}, \mathfrak{I}_{2}\right)$ such that $h\left(x_{1}\right)=x_{2}$. Lemma $3.2(a)$ ends the proof. $(b)$ Let $x_{1}, x_{2} \in X$. As $\left(X, \mathfrak{I}_{1}, \mathfrak{I}_{2}\right)$ is homogeneous, there exists a fuzzy homeomorphism $h:\left(X, \mathfrak{I}_{1}, \mathfrak{I}_{2}\right) \rightarrow\left(X, \mathfrak{J}_{1}, \mathfrak{I}_{2}\right)$ such that $h\left(x_{1}\right)=x_{2}$. Lemma $3.2(b)$ ends the proof. (c) Let $x_{1}, x_{2} \in X$. As $\left(X, \mathfrak{J}_{1}, \mathfrak{J}_{2}\right)$ is homogeneous, there exists a fuzzy homeomorphism $h:\left(X, \mathfrak{I}_{1}, \mathfrak{I}_{2}\right) \rightarrow\left(X, \mathfrak{I}_{1}, \mathfrak{I}_{2}\right)$ such that $h\left(x_{1}\right)=x_{2}$. Therefore, we have

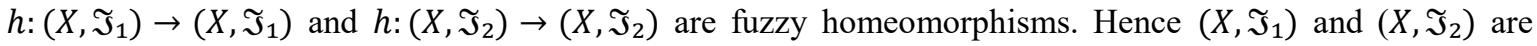
homogeneous. Implication $(a)$ of Theorem 3.3 is not reversible as the following example shows:

Example 3.4. Let $X=\{a, b, c\}, \Im_{1}=\left\{0_{X}, 1_{X}, \mathcal{X}_{\{a\}}\right\}, \mathfrak{J}_{2}=\left\{0_{X}, 1_{X}, \mathcal{X}_{\{b\}}, \mathcal{X}_{\{c\}}, \mathcal{X}_{\{b, c\}}\right\}$. Then $\left\langle\widetilde{J}_{1}, \mathfrak{\Im}_{2}\right\rangle=$ $\left\{X_{Y}: Y \subseteq X\right\}$ and hence $\left(X,<\mathfrak{J}_{1}, \Im_{2}>\right)$ is homogeneous. If $f: X \rightarrow X$ is a bijection for which $f(a)=b$, then we have $X_{\{b, c\}} \in \mathfrak{I}_{1} \cup \mathfrak{I}_{2}$ but $\left.f^{\rightarrow}\left(\mathcal{X}_{\{b, c\}}\right\}\right)=\mathcal{X}_{\left\{a, f^{-1}(c)\right\}} \notin \mathfrak{I}_{1} \cup \mathfrak{I}_{2}$ which shows that $f$ is not a fuzzy pairwise homeomorphism. Hence $\left(X, \mathfrak{J}_{1}, \mathfrak{I}_{2}\right)$ is not pairwise homogeneous.

Implication $(b)$ of Theorem 3.3 is not reversible as the following example shows:

Example 3.5. Let $X=\mathbb{R}, \mathfrak{\Im}_{1}=I^{X}, \mathfrak{I}_{2}=\left\{0_{X}, 1_{X}, \mathcal{X}_{\{2\}}\right\}$. It is not difficult to see that $\left(X, \mathfrak{I}_{1}\right)$ is homogeneous and that $\left(X, \mathfrak{J}_{2}\right)$ is not homogeneous. As $\mathfrak{J}_{1} \cup \mathfrak{I}_{2}=\mathfrak{I}_{1}$, then $\left(X, \mathfrak{\Im}_{1}, \mathfrak{I}_{2}\right)$ is pairwise homogeneous. On the other hand, by Theorem $3.3(c)$, it follows that $\left(X, \mathfrak{J}_{1}, \mathfrak{I}_{2}\right)$ is not homogeneous.

Implication (c) of Theorem 3.3 is not reversible as the following example shows:

Example 3.6. Let $X=\{a, b, c, d, e, f\}, \widetilde{J}_{1}=\left\{X_{Y}: Y \in\{\varnothing, X,\{a, b, c\},\{d, e, f\}\}\right\}$, and $\widetilde{J}_{2}=\left\{X_{Y}: Y \in\right.$ $\{\varnothing, X,\{a, b\},\{c, d\}\},\{e, f\},\{a, b, c, d\},\{a, b, e, f\},\{c, d, e, f\}\}$. It is not difficult to see that $\left(X, \mathfrak{I}_{1}\right)$ is homogeneous, $\left(X, \mathfrak{J}_{2}\right)$ is homogeneous, and $\left(X, \mathfrak{J}_{1}, \mathfrak{I}_{2}\right)$ is not pairwise homogeneous and hence not homogeneous.

Theorem 3.7. Let $\left(X, \widetilde{J}_{1}, \mathfrak{I}_{2}\right)$ be a homogeneous fuzzy bitopological space having a minimal fuzzy open set. If $A \in \mathfrak{I}_{1} \cup \mathfrak{I}_{2}$, then the following are equivalent:

(a) $A$ is an minmal fuzzy open set in $\left(X, \widetilde{\mathfrak{I}}_{1}, \mathfrak{I}_{2}\right)$.

(b) $A$ is a minimal fuzzy open set in $\left(X, \mathfrak{J}_{1}\right)$ or $A$ is a minimal fuzzy open set in $\left(X, \mathfrak{J}_{2}\right)$.

Proof. (a) $\Rightarrow(b)$ Obvious. $(b) \Rightarrow(a)$ Without loss of generality we may assume that $A$ is a minimal fuzzy open set in $\left(X, \widetilde{J}_{1}\right)$. Applying Proposition 1.4, there exists $r_{1} \in(0,1]$ and $Y_{1} \subseteq X$ such that $A=r_{1} x_{Y_{1}}$. Take a minimal fuzzy open set $B$ of $\left(X, \mathfrak{\Im}_{1}, \Im_{2}\right)$. Then again by Proposition 1.4, there exists $r_{2} \in(0,1]$ and $Y_{2} \subseteq X$ such that $B=r_{2} X_{Y_{2}}$. Take $y_{1} \in Y_{1}, y_{2} \in Y_{2}$, and a fuzzy homeomorphism $h:\left(X, \mathfrak{\Im}_{1}, \mathfrak{I}_{2}\right) \rightarrow\left(X, \mathfrak{\Im}_{1}, \mathfrak{\Im}_{2}\right)$ such that $h\left(y_{2}\right)=y_{1}$. Applying Theorem 2.11 (ii), it follows that $h \rightarrow(B)=h^{\rightarrow}\left(r_{2} x_{Y_{2}}\right)=r_{2} x_{h\left(Y_{2}\right)}$ is a minimal fuzzy open set in $\left(X, \mathfrak{\Im}_{1}, \mathfrak{\Im}_{2}\right)$. Since $y_{1} \in Y_{1} \cap h\left(Y_{2}\right)$, then $\left.\left(r_{2} x_{h\left(Y_{2}\right)}\right\}\right) \wedge\left(r_{1} x_{Y_{1}}\right) \neq 0_{X}$. Since $r_{2} x_{h\left(Y_{2}\right)}$ and $r_{1} x_{Y_{1}}$ are minimal fuzzy open sets in $\left(X, \Im_{1}\right)$, then by Corollary 2.7 (ii), it follows that $r_{2} x_{h\left(Y_{2}\right)}=r_{1} x_{Y_{1}}$ and hence $A$ is a minmal fuzzy open set in $\left(X, \mathfrak{J}_{1}, \widetilde{J}_{2}\right)$.

Corollary 3.8. Let $\left(X, \widetilde{J}_{1}, \mathfrak{I}_{2}\right)$ be an homogeneous fuzzy bitopological space having a minimal fuzzy open set and $A \in \mathfrak{I}_{1} \cup \mathfrak{I}_{2}$. Then the following are equivalent:

(a) $A$ is a minimal fuzzy open set in $\left(X, \mathfrak{I}_{1}, \mathfrak{I}_{2}\right)$.

(b) $A$ is a minimal fuzzy open set in $\left(X,\left\langle\mathfrak{I}_{1}, \mathfrak{I}_{2}\right\rangle\right)$.

(c) $A$ is a pairwise minimal fuzzy open set in $\left(X, \mathfrak{J}_{1}, \mathfrak{I}_{2}\right)$.

(d) $A$ is a minimal fuzzy open set in $\left(X, \widetilde{J}_{1}\right)$ or $A$ is a minimal fuzzy open set in $\left(X, \mathfrak{\Im}_{2}\right)$.

(e) $A$ is a minimal fuzzy open set in $\left(X, \mathfrak{J}_{1}\right)$.

$(f) A$ is a minimal fuzzy open set in $\left(X, \mathfrak{J}_{2}\right)$.

Proof. $(a) \Rightarrow(b)$ and $(b) \Rightarrow(c)$ Theorem 2.2. $(c) \Rightarrow(d)$ Corollary 2.9. $(d) \Rightarrow(a)$ Theorem 3.7. $(a) \Leftrightarrow$ (e) and $(a) \Leftrightarrow(f)$ Theorem 3.7.

Theorem 3.9. Let $\left(X, \mathfrak{J}_{1}, \mathfrak{I}_{2}\right)$ be a pairwise homogeneous fuzzy bitopological space. If $A$ is a minimal fuzzy open set in $\left(X, \mathfrak{I}_{1}, \mathfrak{I}_{2}\right)$, then there exist $r \in(0,1]$ and $Y \subseteq X$ such that $A=r \mathcal{X}_{Y}$.

Proof. Suppose on the contrary that there exist two different points $x_{1}, x_{2} \in X$ such that $0<A\left(x_{1}\right)<A\left(x_{2}\right)$. Since $\left(X, \widetilde{\mathfrak{I}}_{1}, \mathfrak{I}_{2}\right)$ is pairwise homogeneous there exists a fuzzy homeomorphism $h:\left(X, \mathfrak{J}_{1}, \mathfrak{I}_{2}\right) \rightarrow\left(X, \mathfrak{J}_{1}, \mathfrak{I}_{2}\right)$ such that $h\left(x_{1}\right)=x_{2}$. As $A$ is pairwise minimal, then by Corollary $2.13 h \rightarrow(A)$ is pairwise minimal. As $(A \wedge$ 
$\left.h^{\rightarrow}(A)\right)\left(x_{2}\right)=\min \left\{A\left(x_{2}\right), A\left(x_{1}\right)\right\}=A\left(x_{1}\right)>0, A \wedge h^{\rightarrow}(A) \neq 0_{X}$. Therefore, by Theorem $2.60_{X}(A)=A$ and hence $A\left(x_{1}\right)=\left(h^{\rightarrow}(A)\right)\left(x_{1}\right)=A\left(x_{2}\right)$, a contradiction.

In the sequel, $\operatorname{GFPH}\left(X, \mathfrak{J}_{1}, \mathfrak{I}_{2}\right)$ will denote the group of all fuzzy pairwise homeomorphisms from $\left(X, \mathfrak{I}_{1}, \mathfrak{I}_{2}\right)$ onto itself. Theorem 3.10. Let $\left(X, \mathfrak{I}_{1}, \mathfrak{I}_{2}\right)$ be a pairwise homogeneous fuzzy bitopological space. If $r X_{Y}$ is a minimal fuzzy open set in $\left(X, \mathfrak{\Im}_{1}, \mathfrak{\Im}_{2}\right)$, then the family $\left\{r X_{h(Y)}: h \in \operatorname{GFPH}\left(X, \mathfrak{\Im}_{1}, \mathfrak{\Im}_{2}\right)\right\}$ is the set of all pairwise minimal fuzzy open sets in $\left(X, \mathfrak{I}_{1}, \mathfrak{I}_{2}\right)$. Proof. Let $A$ be a pairwise minimal fuzzy open set of $\left(X, \mathfrak{\Im}_{1}, \mathfrak{I}_{2}\right)$. Choose $x \in X$ such that $A(x)>0$ and $y \in Y$. As $\left(X, \widetilde{\Im}_{1}, \widetilde{I}_{2}\right)$ is pairwise homogeneous, there exists a fuzzy pairwise homeomorphism $h:\left(X, \mathfrak{\Im}_{1}, \mathfrak{I}_{2}\right) \rightarrow\left(X, \mathfrak{\Im}_{1}, \mathfrak{J}_{2}\right)$ such that $h(y)=x$. Applying corollary 2.13 , it follows that $h^{\leftarrow}(A)$ is pairwise minimal. As $\left(\left(r X_{h(Y)}\right) \wedge h^{\leftarrow}(A)\right)(y)=\min \{A(x), r\}>0, A \wedge$ $h^{\leftarrow}(A) \neq 0_{X}$. Therefore, by Theorem $2.6 h^{\leftarrow}(A)=r X_{Y}$ and hence $A=h^{\rightarrow}\left(r X_{Y}\right)=r X_{h(Y)}$.

Recall that a partition $\mathcal{B}_{X}$ on a nonempty set $X$ is called a regular partition if for any two elements $U, V \in \mathcal{B}_{X},|U|=|V|$. Theorem 3.11. Let $\left(X, \mathfrak{\Im}_{1}, \mathfrak{I}_{2}\right)$ be a pairwise homogeneous fuzzy bitopological space. If $r X_{Y}$ is a minimal fuzzy open set in $\left(X, \mathfrak{\Im}_{1}, \mathfrak{I}_{2}\right)$, then the family $\{h(Y): h \in$ $\left.\operatorname{GFPH}\left(X, \mathfrak{\Im}_{1}, \mathfrak{I}_{2}\right)\right\}$ forms a regular partition on $X$. Proof. It is clear that $h(Y) \neq \varnothing$ for every $h \in$ $\operatorname{GFPH}\left(X, \mathfrak{\Im}_{1}, \mathfrak{\Im}_{2}\right)$. Suppose for some $f, g \in \operatorname{GFPH}\left(X, \mathfrak{\Im}_{1}, \mathfrak{\Im}_{2}\right), f(Y) \cap g(Y) \neq \emptyset$. Choose $x \in f(Y) \cap$ $g(Y), y \in Y$, and $h \in G F P H\left(X, \Im_{1}, \Im_{2}\right)$ such that $h(x)=y$. By Corollary 2.13 , both $(h \circ f) \rightarrow\left(r X_{Y}\right)$ and $(h \circ$ $g) \rightarrow\left(r X_{Y}\right)$ are pairwise minimal fuzzy open sets. Since $\left(\left(r X_{Y}\right) \wedge(h \circ f) \rightarrow\left(r X_{Y}\right) \wedge(h \circ g) \rightarrow\left(r X_{Y}\right)\right)(y)=$ $r>0$ then by Theorem 2.6, it follow that $r X_{Y}=(h \circ f) \rightarrow\left(X_{Y}\right)$ and $r X_{Y}=(h \circ g) \rightarrow\left(r X_{Y}\right)$ and hence $(h \circ f) \rightarrow\left(r X_{Y}\right)=(h \circ g) \rightarrow\left(r X_{Y}\right)$. Thus, $r X_{(h \circ f)(Y)}=r X_{(h \circ g)(Y)}$ and hence $(h \circ f)(Y)=(h \circ g)(Y)$. Therefore, $f(Y)=g(Y)$. To see that $\cup\left\{h(Y): h \in G F P H\left(X, \mathfrak{\Im}_{1}, \mathfrak{\Im}_{2}\right)\right\}=X$, let $z \in X$. Pick $w \in Y$ and $h \in$ $\operatorname{GFPH}\left(X, \mathfrak{I}_{1}, \mathfrak{I}_{2}\right)$ such that $h(w)=z$. Thus, $z \in h(Y)$ which completes the proof.

\section{CUT FUZZY BITOPOLOGICAL SPACES}

Theorem 4.1. If $f:\left(X, \mathfrak{I}_{1}, \mathfrak{I}_{2}\right) \rightarrow\left(Y, \delta_{1}, \delta_{2}\right)$ is fuzzy pairwise continuous (homeomorphism), then for every $a \in[0,1), f:\left(X,\left(\mathfrak{I}_{1}\right)_{a},\left(\mathfrak{J}_{2}\right)_{a}\right) \rightarrow\left(Y,\left(\delta_{1}\right)_{a},\left(\delta_{2}\right)_{a}\right)$ is pairwise continuous (homeomorphism).

Proof. Let $a \in[0,1)$ and let $\left.V \in\left(\delta_{1}\right)_{a} \cup\left(\delta_{2}\right)_{a}\right)$. Then there is $B \in \delta_{1} \cup \delta_{2}$ such that $V=B^{-1}(a, 1]$. Since $f:\left(X, \mathfrak{\Im}_{1}, \mathfrak{I}_{2}\right) \rightarrow\left(Y, \delta_{1}, \delta_{2}\right)$ is fuzzy pairwise continuous, then $f^{\leftarrow}(B) \in \mathfrak{I}_{1} \cup \mathfrak{I}_{2}$ and so $\left(f^{\leftarrow}(B)\right)^{-1}(a, 1] \in$ $\left(\mathfrak{I}_{1}\right)_{a} \cup\left(\mathfrak{I}_{2}\right)_{a}$. Since $f^{-1}(V)=f^{-1}\left(B^{-1}(a, 1]\right)=\left(f^{\leftarrow}(B)\right)^{-1}(a, 1]$, then $f^{-1}(V) \in\left(\mathfrak{I}_{1}\right)_{a} \cup\left(\mathfrak{I}_{2}\right)_{a}$. It follows that $f:\left(X,\left(\mathfrak{J}_{1}\right)_{a},\left(\mathfrak{I}_{2}\right)_{a}\right) \rightarrow\left(Y,\left(\delta_{1}\right)_{a},\left(\delta_{2}\right)_{a}\right)$ is pairwise continuous.

Corollary 4.2. If $\left(X, \mathfrak{I}_{1}, \mathfrak{I}_{2}\right)$ is a pairwise homogeneous fuzzy bitopological space, then for every $a \in[0,1)$ the bitopological space $\left(X,\left(\mathfrak{J}_{1}\right)_{a},\left(\mathfrak{I}_{2}\right)_{a}\right)$ is pairwise homogeneous. The following example shows that the converse of each of Theorems 4.1 and Corollary 4.2 need not to be true in general: Example 4.3. Let

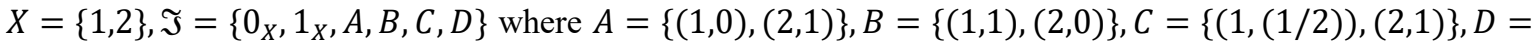
$\{(1,(1 / 2)),(2,0)\}$. Define $h: X \rightarrow X$ by $h(1)=2$ and $h(2)=1$. Then for every $a \in[0,1), h:\left(X, \widetilde{J}_{a}, \widetilde{J}_{a}\right) \rightarrow$ $\left(X, \mathfrak{I}_{a}, \mathfrak{I}_{a}\right)$ is pairwise continuous while $h:(X, \mathfrak{I}, \mathfrak{I}) \rightarrow(X, \mathfrak{I}, \mathfrak{I})$ is not fuzzy pairwise continuous. Therefore, the converse of Theorem 4.1 is not true in general. For every $a \in[0,1), h:\left(X, \mathfrak{J}_{a}, \mathfrak{J}_{a}\right) \rightarrow$ $\left(X, \widetilde{J}_{a}, \widetilde{I}_{a}\right)$ is pairwise homeomorphism with $h(1)=2$ and $h^{-1}(2)=1$ and so $\left(X, \widetilde{J}_{a}, \mathfrak{J}_{a}\right)$ is pairwise homogeneous. On the other hand, it is not difficult to check that $(X, \mathfrak{I}, \mathfrak{I})$ is not pairwise homogeneous. This shows that the converse of Corollary 4.2 is not true in general. Lemma 4.4. [26] If $f:(X, \mathfrak{I}) \rightarrow(X, \mathfrak{I})$ is a fuzzy continuous (homeomorphism) map, then for all a $\in[0,1), f:\left(X, \mathfrak{J}_{a}\right) \rightarrow\left(X, \mathfrak{\Im}_{a}\right)$ is continuous (homeomorphism).

Theorem 4.5. If $f:\left(X, \widetilde{J}_{1}, \widetilde{J}_{2}\right) \rightarrow\left(Y, \delta_{1}, \delta_{2}\right)$ is a fuzzy continuous (homeomorphism) function, then for all $a, b \in[0,1), f:\left(X,\left(\mathfrak{J}_{1}\right)_{a},\left(\mathfrak{J}_{2}\right)_{b}\right) \rightarrow\left(Y,\left(\delta_{1}\right)_{a},\left(\delta_{2}\right)_{b}\right)$ is continuous (homeomorphism). Proof. We prove only the continuity part. Suppose that $f:\left(X, \widetilde{J}_{1}, \mathfrak{J}_{2}\right) \rightarrow\left(Y, \delta_{1}, \delta_{2}\right)$ is a fuzzy continuous function. Then both $f:\left(X, \mathfrak{J}_{1}\right) \rightarrow\left(Y, \delta_{1}\right)$ and $f:\left(X, \Im_{2}\right) \rightarrow\left(Y, \delta_{2}\right)$ are fuzzy continuous. Therefore by Lemma 4.4, $f:\left(X, \widetilde{\Im}_{a}\right) \rightarrow\left(X, \widetilde{I}_{a}\right)$ and $f:\left(X, \mathfrak{I}_{b}\right) \rightarrow\left(X, \mathfrak{\Im}_{b}\right)$ are continuous. It follows that $f:\left(X,\left(\mathfrak{I}_{1}\right)_{a},\left(\mathfrak{I}_{2}\right)_{b}\right) \rightarrow$ $\left(Y,\left(\delta_{1}\right)_{a},\left(\delta_{2}\right)_{b}\right)$ is continuous. Corollary 4.6. If $\left(X, \mathfrak{I}_{1}, \mathfrak{I}_{2}\right)$ is a homogeneous fuzzy bitopological space, then for all $a, b \in[0,1)$ the bitopological space $\left(X,\left(\mathfrak{I}_{1}\right)_{a},\left(\mathfrak{I}_{2}\right)_{b}\right)$ is homogeneous. The following example will show that the converse of Corollary 4.6 need not to be true in general:

Example 4.7. Let $X=\{1,2\}$ and

$$
\mathfrak{J}=\{B: B(X) \subseteq[0,(1 / 2)]\} \cup\{B: B(X) \subseteq[(1 / 2), 1]\} \cup\{B: B(1) \leq B(2)\} .
$$

It is not difficult to check that $\mathfrak{\Im}$ is a fuzzy topology on $X$ and that $\mathfrak{J}_{a}$ is the discrete topology on $X$ for all $a \in[0,1)$. Thus, we have $\left(X, \widetilde{J}_{a}, \mathfrak{I}_{b}\right)$ is a homogeneous bitopological space for all $a, b \in[0,1)$. On the other hand, if $(X, \mathfrak{I}, \mathfrak{I})$ is a homogeneous fuzzy bitopological space, then there is a fuzzy homeomorphism 
$h:(X, \mathfrak{I}, \mathfrak{I}) \rightarrow(X, \mathfrak{I}, \mathfrak{I})$ such that $h(1)=2$. So $h:(X, \mathfrak{I}) \rightarrow(X, \mathfrak{I})$ is a fuzzy homeomorphism. Let $B=\{(1,0),(2,1)\}$, then $B \in \mathfrak{I}$ and so $h^{\leftarrow}(B) \in \mathfrak{I}$. But $h^{\leftarrow}(B)=B \circ h=\{(1,1),(2,0)\} \notin \mathfrak{I}$. It follows that $(X, \mathfrak{I}, \mathfrak{I})$ is not homogeneous.

\section{REFERENCES}

[1] L. A. Zadeh, "Fuzzy Sets," Inform and control, vol. 8, pp. 338-353, 1965.

[2] C. K. Wong, "Fuzzy Points and Local Properties of Fuzzy Topology," Journal of Mathematical Analysis and Applications, vol. 46, pp. 316-328, 1974.

[3] M. D. Weiss, "Fixed Points, Separation, and Induced Topologies for Fuzzy Sets," Journal of Mathematical Analysis and Applications, vol. 50, pp. 142-150, 1975.

[4] C. L. Chang, "Fuzzy Topological Spaces," Journal of Mathematical Analysis and Applications, vol. 24, pp. 182190, 1968.

[5] J. C. Kelly, "Bitopological Spaces," Proceedings of the London Mathematical Society, vol. 13, pp. 71--89, 1963.

[6] A. Kandil, "Biproximities and Fuzzy Titopological Spaces," Simon Stevin, vol. 63, pp. 45-66, 1989.

[7] S. Debnath, "On Fuzzy Biminimal Structure Spaces," International Journal of Mathematical Analysis, vol. 6, pp. 841--845, 2012.

[8] B. C. Tripathy, S. Debnath, " $\gamma$-Open Sets and $\gamma$-Continuous Mappings in Fuzzy Bitopological Spaces," Journal of Intelligent \& Fuzzy Systems, vol. 24, pp. 631--635, 2013

[9] B. C. Tripathy, S. Debnath, "On Fuzzy b-Locally Open Sets in Bitopological Spaces," Songklanakarin Journal of Science and Technology, vol. 37, pp. 93-96, 2015.

[10] A. Fora, S. Al Ghour, "Homogeneity in Fuzzy Spaces," Questions and Answers in General Topology, vol. 19, pp.159--164, 2001.

[11] F. Nakaoka, N. Oda, "Some Applications of Minimal Fuzzy Open Sets," International Journal of Mathematics and Mathematical Sciences, vol. 27, pp. 471--476, 2001.

[12] S. Al Ghour, A. Fora, "Minimality and Homogeneity in Fuzzy Spaces," Journal of Fuzzy Mathematics, vol. 12, pp. 725--737, 2004.

[13] S. Al Ghour, "Some Generalizations of Minimal Fuzzy Open Sets," Acta Mathematica Universitatis Comenianae, vol. 75, pp. 107--117, 2006.

[14] S. Al Ghour, "On Mximal and Minimal Fuzzy Sets in I-Topological Spaces," International Journal of Mathematics and Mathematical Sciences, Art. ID 180196, 11 pp, 2010.

[15] S. Al Ghour, A. Azaizeh, "Minimal Open Sets in Bitopological Spaces," Questions and Answers in General Topology, vol. 29, pp. 105-112, 2011.

[16] R. Lowen, "A Comparison of Different Compactness Notions in Fuzzy Topological Spaces," Journal of Mathematical Analysis and its Applications, vol. 64, pp. 446--454, 1978.

[17] S. E. Rodabaugh, "The Hausdorff Separation Axiom for Fuzzy Topological Spaces," Topology and its Applications., vol. 11, pp. 319--334, 1980.

[18] A. J. Klein, "a-Closure in Fuzzy Topology," Rocky Mountain J. Math., vol. 11, pp. 553--560, 1981.

[19] S. E. Rodabaugh, "A Categorical Acommodation of Various Notions of Fuzzy Topology," Fuzzy Sets and Systems, vol. 9, pp. 241--265, 1983.

[20] P. Wuyts, "On the Determination of Fuzzy Topological Spaces and Fuzzy Neighbourhood Spaces by Their LevelTopologies," Fuzzy Sets and Systems, vol. 12, pp. 71--85, 1984.

[21] S. E. Rodabaugh, R. Lowen, "Para-Lowen, and $\alpha$-Level Functors and Fuzzy Topologies on the Crisp Real Line," Journal of Mathematical Analysis and Applications, vol. 131, pp. 157--169, 1988.

[22] Y. C. Bai, Y. L. Zheng, "Type II Strong Fuzzy Paracompactness and the $\lambda$-Cut Topology," Pure and Applied Mathematics, vol. 11, pp. 139--142, 1995.

[23] Y. L. Zheng, X. C. Du, "ג-Cut Topologies of L-Fuzzy Topologies," J. Baoji College Arts Sci. Nat. Sci., vol. 16, pp. $1--3,1996$.

[24] P. Wuyts, "On Level-Topologies and Maximality of Fuzzy Topological Spaces," Fuzzy Sets and Systems, vol. 79, pp. 337--339, 1996.

[25] S. S. Benchalli, G. P. Siddapur, "On the Level Spaces of Fuzzy Topological Spaces," Bulletin of Mathematical Analysis and Applications, vol. 1, pp. 57--65, 2009.

[26] S. Al Ghour, A. Fora, "On CDH Fuzzy Spaces," Journal of Intelligent \& Fuzzy Systems, vol. 30, pp. 935-941, 2016.

[27] A. K. Srivastava, S. P. Tiwari, "On Relationships Among Fuzzy Approximation Operators, fuzzy topology, and fuzzy automata," Fuzzy Sets and Systems, vol. 138, pp. 197--204, 2003.

[28] A. K. Srivastava, S. P. Tiwari, "IF-Topologies and IF-Automata," Soft Computing, vol. 14, pp. 571-578, 2010.

[29] M. Horry, M. M. Zahedi, "Some (Fuzzy) Topologies on General Fuzzy Automata," Iranian Journal of Fuzzy Mathematics, Vol. 10, pp. 73.89. 